\title{
Transsexualism: A Different Viewpoint to Brain Changes
}

\author{
Mohammad Reza Mohammadi, Ali Khaleghi \\ Psychiatry \& Psychology Research Center, Tehran University of Medical Sciences, Tehran, Iran
}

\begin{abstract}
Transsexualism refers to a condition or belief which results in gender dysphoria in individuals and makes them insist that their biological gender is different from their psychological and experienced gender. Although the etiology of gender dysphoria (or transsexualism) is still unknown, different neuroimaging studies show that structural and functional changes of the brain result from this sexual incongruence. The question here is whether these reported changes form part of the etiology of transsexualism or themselves result from transsexualism culture, behaviors and lifestyle. Responding to this question can be more precise by consideration of cultural neuroscience concepts, particularly the culture-behaviorbrain (CBB) loop model and the interactions between behavior, culture and brain. In this article, we first review the studies on the brain of transgender people and then we will discuss the validity of this claim based on the CBB loop model. In summary, transgender individuals experience change in lifestyle, context of beliefs and concepts and, as a result, their culture and behaviors. Given the close relationship and interaction between culture, behavior and brain, the individual's brain adapts itself to the new condition (culture) and concepts and starts to alter its function and structure.
\end{abstract}

KEY WORDS: Transsexualism; Gender dysphoria; Gender identity; Cultural neuroscience; Human brain; Behavior.

\section{INTRODUCTION}

The evaluation of the neuropsychological and neurophysiological differences between males and females has been a hot topic among neuroscientists, because various structural and functional differences of the human brain have been observed in a sex dependent manner. For instance, differences have been reported in the whole brain, cortical thickness (CTh), lobar volumes, grey and white matter volumes, corpus callosum size and connectivity profiles, and it is shown that they are correlated with distinctive cognitive abilities of males and females regarding visuospatial processing and language. ${ }^{1-8)}$ Motivated by this category of studies, many researchers have attempted to investigate the human brain in the gender identity disorder or more recently in gender dysphoria (GD) in the past few years (actually, GD replaced gender identity dis-

Received: December 15, 2017 / Revised: February 12, 2018

Accepted: February 13, 2018

Address for correspondence: Ali Khaleghi, PhD

Psychiatry \& Psychology Research Center, Tehran University of

Medical Sciences, Tehran 1333795914, Iran

Tel: +98-9163464494, Fax: +98-21-55421959

E-mail: alikhaleghi_bme84@yahoo.com

ORCID: https://orcid.org/0000-0002-9035-7075 order since the publication of the 5th edition of the DSM in 2013). Individuals with GD are called transgender and they often feel an emotional and social pressure. Transsexualism refers to a condition or belief which results in GD in individuals and makes them insist that their biological gender is different from their psychological and experienced gender. GD or gender incongruence is usually accompanied by distress, and, consequently, transgender people often look for genital surgical procedure and cross-sex hormonal treatment in order to align their biological gender to their experienced one. Here, we will use transgender as a general term to refer to both trans-men (female-to-male, FtM) and trans-women (male-to-female, $\mathrm{MtF})$.

Although the etiology of GD is still unknown, environmental and biological factors have been argued to contribute to the variations of gender identity. ${ }^{9-13)}$ Studies on twins, ${ }^{14,15)}$ birth order, ${ }^{16,17)}$ familial groups ${ }^{18)}$ and sex genes $^{19,20)}$ have supported biological causes, such as prenatal exposure to sexual hormones and genetic factors, for GD. These studies are sparse and their results are sometimes inconsistent. Some studies have reported no obvious biological incongruities, such as sexual chromo-

(ㄷ) This is an Open-Access article distributed under the terms of the Creative Commons Attribution Non-Commercial License (http://creativecommons.org/licenses/by-nc/4.0) which permits unrestricted non-commercial use, distribution, and reproduction in any medium, provided the original work is properly cited. 
some aberrations, in transgender individuals ${ }^{21)}$; some other studies have suggested a possible association between genetic factors and only one type of the transsexulaism, for example a higher risk for MtF transsexulism among non-twin siblings ${ }^{18)}$ or the assumption of prenatal testosterone exposure for MtF transsexulism, ${ }^{22-24)}$ and the association between polymorphism of the CYP17, particularly CYP17-34C, gene and FtM but not MtF transsexualism. ${ }^{25)}$ Also, some existing literature from this category of studies is based on different case reports and they need to be confirmed by more accurate study designs. Moreover, studies on sexual hormone related genes need to be replicated with a larger population of transgender people and multiple testing corrections should be implemented to obtain solid results. For these reasons, two review studies on sexual orientation and transsexualism (as well as homosexualism) suggested that no clear and distinct genetic relationship with transexualism and sexual orientation has been observed up to the present time. ${ }^{26,27)}$

In relation to the etiology of GD, we should consider other potential mental problems and environmental factors. Review the literature on comorbid psychiatric conditions of GD demonstrates that different estimated prevalence of comorbid psychiatric disorders have been reported in individuals with GD. ${ }^{28)}$ A recent study reported current prevalence of comorbid conditions (according to another current DSM disorders other than personality disorders) in $38 \%$ of MtFs and $37 \%$ of FtMs. This study also reported lifetime prevalence of DSM disorders, excluding personality disorders, in $68 \%$ of MtFs and $71 \%$ of FtMs. ${ }^{29)}$ Personality disorders have been observed in $12 \%$ of MtFs and $18 \%$ of FtMs. Most prevalent personality disorders in transgender people were schizoid, avoidant and borderline. ${ }^{29)}$ Moreover, regarding environmental factors, parenting and family variables, such as very closeness to the mother, father absence, parental wish for a boy or daughter and psychopathology in the parents, have been suggested to contribute to the GD as major factors. ${ }^{9)}$ Also, wrong self-knowledge is an another potential contributing factor to the GD, which means the wrong recognition of the natal sex by the subject independent from parenting due to improper experiences in life and misunderstanding of these experiences. ${ }^{30)}$

It is suggested that the divergence between natal sex and gender identity arises from the temporal contrast between sexual differentiations of the genital organs and the brain. ${ }^{31,32)}$ In fact, the biological influence of testosterone or absence of this hormone during the first half of pregnancy results in development of male or female genital organs, respectively. However, the brain differentiates sexually during the second half of pregnancy, which is much later than that of sexual organs in the course of fetal development. Therefore, these two developmental processes are chronologically independent, and it is concluded that masculinization or feminization of the genitals may not inevitably bring into that of the brain. For this reason, several neurophysiological and neuroimaging studies have investigated structural and functional differences of the brain between transgender and non-transgender people to prove this claim. In the following, we first review the studies on the brain of transgender people and then we will discuss the validity of this claim.

\section{MAIN SUBJECTS}

\section{Brain Characteristics of Transgender People}

\section{Cortical thickness}

A few studies have examined regional CTh of transgender people to provide more insight into the neurobiology of sexual orientation. Zubiaurre-Elorza et al. ${ }^{33)}$ investigated $C T h$ patterns in untreated transgender individuals. They reported larger CTh of FtM transgenders in the temporal and parietal cortices compared to control males. However, they found no differences between FtM transgenders and control females in terms of CTh. MtF transgenders had larger CTh than males in the insular, orbitofrontal and medial occipital regions. The authors concluded that their evidence demonstrated CTh feminization for MtF. Luders et al. ${ }^{34)}$ reported thicker cortices in untreated $\mathrm{MtF}$ transgenders compared to control males in the both left and right hemispheres, including frontal, temporal and parietal cortices, central sulcus and paracentral gyrus. In fact, they supported CTh feminization for MtF transgenders. Besides these, Abé et al. ${ }^{35)}$ investigated CTh in homosexual men compared to control males and females. They reported thinner visual cortex and smaller thalamus volumes in homosexuals compared to control males.

\section{White matter}

Corpus callosum, as largest structure of white matter in 
the human brain that connects the right and left hemispheres and communicates between them, is a target region in the brain for comparing transgender and nontransgender people. Emory et al.. ${ }^{36)}$ reported no significant differences in callosal shape between transgender individuals and control men and women. However, Yokota et al. ${ }^{37)}$ found that transgender people were more likely to their mental sex than to their natal sex in terms of callosal shape at the midsagittal plane. Furthermore, white matter morphometry has been analyzed using diffusion tensor imaging (DTI) to examine fractional anisotropy (FA), as a measure of white matter microstructure, in transgenders. Using this measure, Rametti et al. ${ }^{38)}$ investigated the white matter microstructure pattern of transgender individuals, who declared early-onset gender incongruence and homosexual orientation, and observed that FtM transgenders had larger FA values than that of control females in the right superior longitudinal fasciculus, corticospinal tract and the forceps minor. Also, they showed lower FA values compared to control males in the corticospinal tract. The authors concluded that the white matter microstructure pattern of FtM transgenders was closer to that of their gender identity (males) than that of their natal sex (females), and there were evidences for intrinsic contrast in the brain structure of untreated FtM transgenders. Moreover, this research group reported significant differences between MtF transgenders and both male and female controls in the superior longitudinal fasciculus, corticospinal tract, forceps minor and anterior cingulum. ${ }^{39)}$ They suggested that some fascicles do not complete the masculinization of the brain in MtF transgenders. In another study, Kranz et al. ${ }^{40)}$ investigated the white matter microstructure pattern of untreated transgender individuals with early-onset gender incongruence using DTI. The authors found extensive significant differences in mean diffusivity between transgenders and male and female controls in most white matter tracts. However, no significant differences in FA have reported in this study.

\section{Grey matter}

Several studies have focused on grey matter as a major part of the central nervous system to investigate transgenders' brain. The results of these studies showed that size and neuron population of the hypothalamic uncinate nucleus (subnucleus 3) and bed nucleus of the stria terminalis in MtF transgenders were similar to control fe- males. ${ }^{41-43)}$ Two another studies found no differences in grey matter proportions between untreated MtF transgenders and control males. ${ }^{44,45)}$ Although Simon et al. ${ }^{46)}$ found significant differences between transgender subjects and control males and females in terms of grey matter volumes in several brain regions, most of these structural differences were dependent on the natal sex rather than gender identity. Putamen volume is another structure of grey matter that has investigated in several researches on transgenders' brain. Two studies reported that the putamen of transgenders was similar to their gender identity, ${ }^{33,44)}$ whereas another study did not confirm their findings and reported contrary results in transgenders. ${ }^{45)}$ The findings on the cerebellum are also inconsistent. Savic and Arver $^{45)}$ found larger grey matter volume within the cerebellum in $\mathrm{MtF}$ transgenders compared to control females, whereas Simon et al. ${ }^{46)}$ found smaller grey matter volume within the cerebellum in MtF transgenders.

Structural and functional connectivity profiles

Hahn et al. ${ }^{47)}$ investigated structural connectivity of untreated transgenders using diffusion weighted images. They reported a decreased intrahemispheric lobar connectivity weights between cortical and subcortical/limbic regions in FtM transgenders and increased interhemispheric lobar connectivity in $\mathrm{MtF}$ transgenders compared to control females and males. Santarnecchi et al. ${ }^{48)}$ investigated functional connectivity of an untreated FtM transgender using resting-state fMRI. They observed a connectivity profile similar to her natal sex rather than gender identity for this FtM. Lin et al. ${ }^{30)}$ examined the degree centrality of the resting-state functional connectivity network in transgenders compared to cissexuals. They reported higher degree centrality in the primary somatosensory cortex and the bilateral superior parietal lobule for transgender subjects. Also, they found that the connectivity profile of transgenders between the bilateral primary somatosensory cortices and the right insula had a negative correlation with the selfness rating of their gender identity.

\section{Task-related neuroimaging findings}

Several neuroimaging studies investigated the functional pattern of transgenders' brain during task performance. Berglund et al. ${ }^{49)}$ found that MtF transgenders exhibited a hypothalamic activation similar to control females when smelling odorous steroids. Burke et al. ${ }^{50)}$ 
found that adolescents with GD exhibited a hypothalamus response to androstadienone similar to their gender identity. The authors observed less clear findings in children; that is, boys with GD showed an activation pattern similar to their biological sex, while girls with GD showed a different activation pattern of the hypothalamus from controls. Ku et al. ${ }^{51)}$ and Gizewski et al. ${ }^{52)}$ investigated functional patterns in transgenders when watching erotic videos. They found that brain activation patterns of MtF transgenders were similar to control females. Soleman et al. ${ }^{53)}$ found underactivation of the superior temporal lobe in FtM transgenders compared to control females during processing of positive affective pictures. Junger et al. ${ }^{54)}$ found significant differences in the precuneus, the insula and the medial prefrontal gyrus in MtF transgenders compared to control males and females during responding to male versus female voices. The authors suggested an intermediate position between male and female for $\mathrm{MtF}$ transgender subjects. Schöning et al. ${ }^{55)}$ reported significant differences in the left parietal and the temporaloccipital regions in $\mathrm{MtF}$ transgenders compared to control males when subjects performed a visuospatial task. However, Soleman et al., ${ }^{56)}$ in another study, investigated brain activation of adolescents with GD and healthy controls when performing a semantic and phonetic verbal fluency task. They did not found significant differences in neuronal activation between adolescents with and without GD. Staphorsius et al. ${ }^{57)}$ also found no significant sex differences in untreated adolescents with GD when performing an executive function task.

In general, an overview of brain morphometric and neural activity findings in transgenders demonstrates contradictory results, which cannot reflect masculinization or feminization of the transgenders' brain. Indeed, there are very inconsistent neuroanatomical and neurofunctional differences between transgender subjects and same sex controls that are detectable at the group level rather than individual level. The question here is whether these reported changes form part of the etiology of transsexualism or themselves result from transsexualism culture, behaviors and lifestyle. Responding to this question can be more precise by consideration of cultural neuroscience concepts, particularly a culture-behavior-brain (CBB) model and the interactions between behavior, culture and brain. Therefore, here, we introduce the CBB loop model and its different aspects. In the following, we propose a hypoth- esis that may justify changes in the function and structure of the transgender's brain, and provide us with a better understanding of the etiology of GD and the way to handle different treatment approaches for GD.

\section{The CBB Loop Model of Human Development}

The CBB loop model assumes that original concepts are originated from the individual level and are disseminated in a population level via social interactions. ${ }^{58)}$ Then, these novel concepts become dominant shared ideas, beliefs, values and behavioral scripts that affect and contextualize human behavior. It is shown that the structural and functional organization of the brain changes as a result of accepting cultural beliefs and performing culturally patterned behaviors due to inherent neuroplasticity. ${ }^{58-61)}$ Then, the modified brain directs individual behaviors for fitting into specific cultural concepts and adjusts concurrent sociocultural environments. The CBB loop model (Fig. 1) suggests two types of behaviors: (1) Culturally contextualized behavior indicates observed actions that are controlled by a specific cultural concept to a large extent.

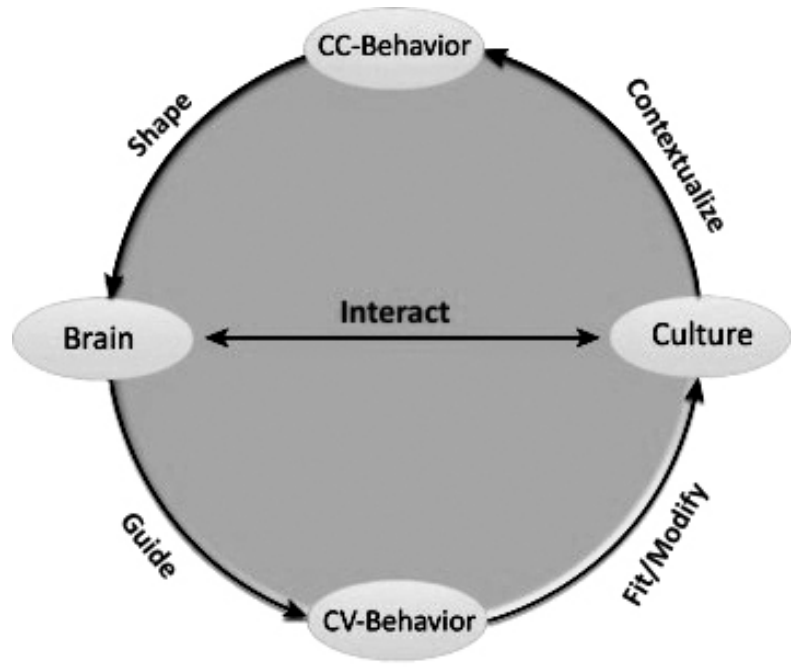

Fig. 1. Illustration of the culture-behavior-brain (CBB) loop model of human development. Cultural environments contextualize human behaviors. Learning novel cultural beliefs and the practice of different behavioral scripts in turn modify the functional organization of the brain. The modified brain then guides individual behavior to voluntarily fit into a cultural context and meanwhile to modify current cultural environments. Direct interactions also occur between culture and brain without overt behavior.

CC-Behavior, culturally contextualized behavior; CV-Behavior, culturally voluntary behavior.

Reused from the article of Han and Ma (Trends Cogn Sci 2015;19: $666-676)^{58)}$ with original copyright holder's permission. 
This type of behavior may not take place when giving up a specific cultural environment. (2) Culturally voluntary behavior refers to observed actions that are controlled by specific cultural beliefs and behavioral scripts that are embedded in the brain as a result of exposure to a specific cultural environment. If the cultural system within the brain remains stable to a certain extent, then the culturally voluntary behavior may take place independently of a specific cultural concept. The CBB loop model also suggests two types of culture-brain interactions: (1) behavior-mediated interaction between culture and brain through observed behavioral practice, and (2) direct interaction between culture and brain with no observed actions. In fact, culture, behavior and the brain interact dynamically as three key factors of the CBB loop model through mutual connections. Each factor and connection of the CBB loop changes continually with time and influences human ontogeny. However, the fundamental role of the genes cannot be ignored in this loop model. Genes lay the groundwork for the structure and function of the brain, culturally voluntary behaviors and culturally contextualized behaviors, as shown in Figure 2 .

\section{Our Hypothesis about Brain Changes in Transgenders Based on the CBB Loop Model}

The CBB loop model enables us to hypothesize on future changes of the brain by taking into account the current culturally patterned behavior and the relevant brain function. Furthermore, we may predict that related brain changes result in human adaptation to specific sociocultural environments and new beliefs and behaviors, i.e., a new lifestyle. Here, based on this concept, we see the reported changes in the transgenders' brain with a new approach. A review of genetic studies on sexual orientation and transsexualism (as well as homosexualism) reveals that no clear and distinct genetic relationship with transexualism and sexual orientation has been observed up to the present time. ${ }^{26,27)}$ Therefore, it might be expected that the changes that occur in the brain of transgender individuals start to appear after a particular stage and period of life. This particular stage probably starts from the time when the child favors toys that are not suitable for his/her gender, or when he/she likes to play with kids of the opposite sex alone, or when he/she is brought up in a family that does not accept his/her gender and shows biased interests in the opposite sex and treats the child with behaviors appropriate for the opposite sex.

Existing literature demonstrates that there is no solid evidence for the differences in the function/structure of the brain between children and adolescents with and without GD. Indeed, there is no comprehensive selfness and understanding of sexual issues in childhood and adolescence. The cultural and sociocultural contexts and specific cultural environment based behaviors have not yet fully developed in this period. In fact, these contexts are emerging and developing in this period of life. Therefore, it seems reasonable to expect that at this age, major behavioral changes have not formed at the social level and, consequently, the brain has not undergone significant changes. After adolescence and over time, the subject becomes more familiar with gender and sexual is-

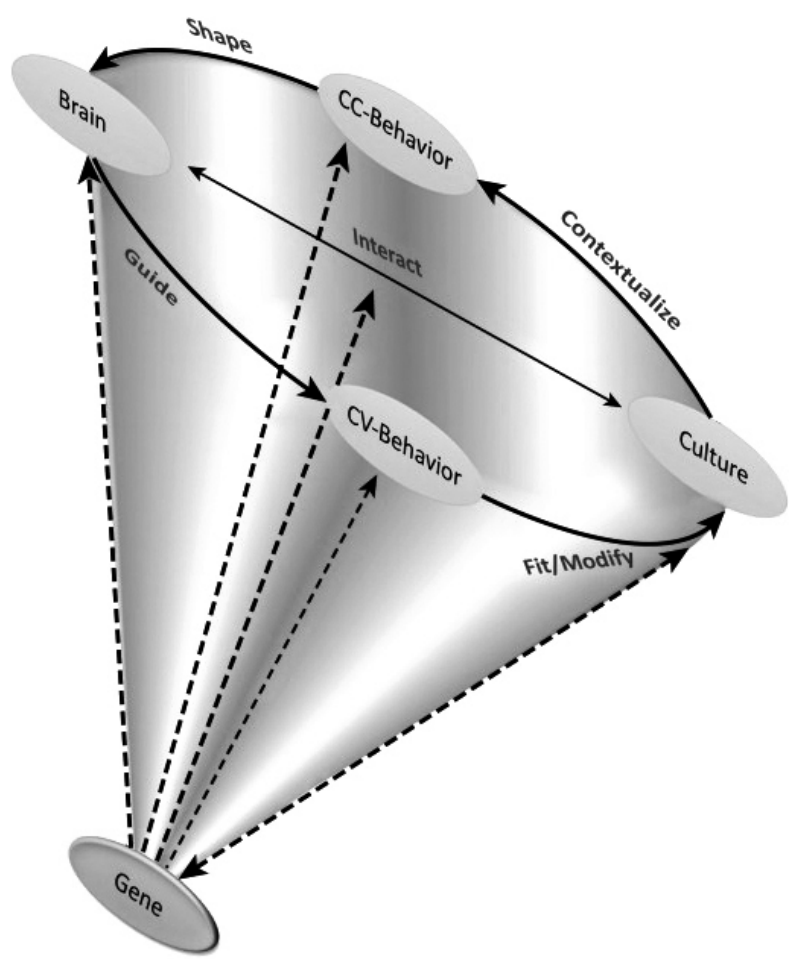

Fig. 2. Genes provide a fundamental basis for the culture-behaviorbrain (CBB) loop in several ways, including genetic influences on the brain and behavior, mutual interactions between genes and culture, and genetic moderations of the association between brain and culture. The unbroken lines in the CBB loop indicate fast interactions between two nodes, whereas the broken lines linking genes and the CBB loop indicate slow interactions between genes and the CBB loop.

CC-Behavior, culturally contextualized behavior; CV-Behavior, culturally voluntary behavior.

Reused from the article of Han and Ma (Trends Cogn Sci 2015;19: $666-676)^{58)}$ with original copyright holder's permission. 
sues and faces transsexualism (or even homosexuality) due to lack of proper selfness, lack of appropriate parenting or his/her childhood background. Then, new ideas and beliefs (i.e., a new specific cultural environment) form for the subject that lead to inappropriate for his/her own biological sex. Consequently, the subject attempts to avoid right and normal culturally contextualized behaviors. He/she imagines himself/herself in his/her preferred gender identity rather than his/her biological sex, and thus tries to adapt his/her values, lifestyle and behaviors to his/her gender identity. Therefore, a subject with GD tries to experience life in his/her preferred gender identity and skews from his/her biological gender and normal culturally contextualized behaviors every day. Depending on how much the subject with GD persists in changing his/her gender (i.e., positive, neutral or negative transgenders) and the extent to which he/she has experienced gender identity, we will observe brain changes and, as a result, related culturally voluntary behaviors. Perhaps this is why the results of brain studies in transgender people are largely contradictory. However, for instance, when a person with GD whose biological gender is male, regulates his lifestyle based on his preferred gender identity (i.e., the female), we should expect, according to the CBB loop model, the brain to adapt to this belief and this lifestyle with time, and consequently changes in function and then the structure of the brain occur. This applies to transgender people with a female biological gender and a male's gender identity. For example, as mentioned earlier, males with GD exhibited functional patterns of the brain similar to control females when watching erotic videos $^{51,52)}$; or some evidence showed CTh feminization for the males with GD. ${ }^{33)}$ Also, females with GD exhibited activation patterns of the brain similar to control males during the processing of positive affective pictures ${ }^{53)}$; or some evidence showed masculinization of the white matter microstructure pattern for females with GD. ${ }^{33)}$

On the other hand, after adaptation of the brain, or more precisely a part of the brain, with gender identity, in addition to the culturally voluntary behaviors, the subject with GD still has not completely abandoned some of the culturally contextualized behaviors derived from habits, family and community environments and so on. In other words, he/she still has innate biological genitals and is recognized with his/her biological sex in the family and society. Therefore, a transgender subject encounters many contradictions and conflicts in his/her personal and social life. This situation leads a transgender subject to different psychological and psychiatric problems.

\section{CONCLUSION}

In summary, transgender individuals experience change in lifestyle, context of beliefs and concepts and, as a result, their culture and behaviors. Given the close relationship and interaction between culture, behavior and brain, the individual's brain adapts itself to the new condition (culture) and concepts and starts to alter its function and structure. In fact, the individual has distanced from the specific cultural environment and so does not exhibit culturally contextualized behaviors any more. Therefore, the function and structure of the brain become affected after a while (the certain period during which the cultural environment is undergoing a change) and drive the individual toward culturally voluntary behaviors, followed by changes in the cultural environment and the individual's lifestyle. Thus, we believe that after a certain period of management, transgender individuals can be driven toward culturally contextualized behaviors via changing the specific cultural environment and lifestyle and can be adapted to their original biological gender through trying to enjoy a natural sexual relationship. Afterward, the brain again will adapt to normal initial conditions based on its own biological sex and modify and fit itself toward the same-sex brain state.

\section{- Acknowledgments}

The authors wish to acknowledge and appreciate the editorial changes made to the manuscript by Fatemeh Daftari (Department of English, Tehran University).

\section{REFERENCES}

1. Gur RC, Turetsky BI, Matsui M, Yan M, Bilker W, Hughett P, et al. Sex differences in brain gray and white matter in healthy young adults: correlations with cognitive performance. I Neurosci 1999;19:4065-4072.

2. Allen JS, Damasio H, Grabowski TJ. Normal neuroanatomical variation in the human brain: an MRI-volumetric study. Am J Phys Anthropol 2002;118:341-358.

3. Leonard CM, Towler S, Welcome S, Halderman LK, Otto R, Eckert MA, et al. Size matters: cerebral volume influences sex differences in neuroanatomy. Cereb Cortex 2008;18:29202931. 
4. Brun CC, Leporé N, Luders E, Chou YY, Madsen SK, Toga AW, et al. Sex differences in brain structure in auditory and cingulate regions. Neuroreport 2009;20:930-935.

5. Giedd JN, Raznahan A, Mills KL, Lenroot RK. Review: magnetic resonance imaging of male/female differences in human adolescent brain anatomy. Biol Sex Differ 2012;3:19.

6. Inano S, Takao H, Hayashi N, Yoshioka N, Mori H, Kunimatsu $\mathrm{A}$, et al. Effects of age and gender on neuroanatomical volumes. J Magn Reson Imaging 2013;37:1072-1076.

7. Kim SW, Lee JY, Kang HJ, Kim SY, Bae KY, Kim JM, et al. Gender-specific associations of the brain-derived neurotrophic factor Val66Met polymorphism with neurocognitive and clinical features in schizophrenia. Clin Psychopharmacol Neurosci 2016;14:270-278.

8. Swapnajeet S, Subodh B, Gourav G. Modafinil dependence and hypersexuality: a case report and review of the evidence. Clin Psychopharmacol Neurosci 2016;14:402-404.

9. Cohen-Kettenis PT, Gooren LJ. Transsexualism: a review of etiology, diagnosis and treatment. J Psychosom Res 1999;46: 315-333.

10. Dawood K, Bailey JM, Martin NG. Genetic and environmental influences on sexual orientation. In: Kim YK, editor. Handbook of behavior genetics. New York:Springer;2009. p.269-279.

11. Steensma TD, McGuire JK, Kreukels BP, Beekman AJ, Cohen-Kettenis PT. Factors associated with desistence and persistence of childhood gender dysphoria: a quantitative follow-up study. J Am Acad Child Adolesc Psychiatry 2013;52: 582-590.

12. Ohadi B, Mohammadi M, Rahimian E, Raisi F, Saberi M, Arbabi $\mathrm{M}$, et al. Brain imaging characteristics in transsexual and normal individuals. J Adv Cognit Sci 2007;9:20-25.

13. Khodayarifard M, Mohammadi MR, Abedini Y. Cognitive-behavioral therapy with emphasis on spiritual therapy in treatment of transsexualism: a case study. Iran J Psychiatry Clin Psychol 2004;9:12-21.

14. Zucker KJ, Bradley SJ. Gender identity disorder and psychosexual problems in children and adolescents. New York: Guilford Press; 1995.

15. Heylens G, De Cuypere G, Zucker KJ, Schelfaut C, Elaut E, Vanden Bossche $\mathrm{H}$, et al. Gender identity disorder in twins: a review of the case report literature. I Sex Med 2012;9: 751-757.

16. Gómez-Gil E, Esteva I, Carrasco R, Almaraz MC, Pasaro E, Salamero M, et al. Birth order and ratio of brothers to sisters in Spanish transsexuals. Arch Sex Behav 2011;40:505-510.

17. VanderLaan DP, Blanchard R, Wood H, Garzon LC, Zucker $\mathrm{KJ}$. Birth weight and two possible types of maternal effects on male sexual orientation: a clinical study of children and adolescents referred to a Gender Identity Service. Dev Psychobiol 2015; 57:25-34.

18. Gómez-Gil E, Esteva I, Almaraz MC, Pasaro E, Segovia S, Guillamon A. Familiality of gender identity disorder in non-twin siblings. Arch Sex Behav 2010;39:546-552.

19. Fernández R, Esteva I, Gómez-Gil E, Rumbo T, Almaraz MC, Roda $\mathrm{E}$, et al. Association study of ER $\beta, A R$, and CYP19A1 genes and MtF transsexualism. J Sex Med 2014;11:29862994.

20. Fernández R, Esteva I, Gómez-Gil E, Rumbo T, Almaraz MC, Roda $\mathrm{E}$, et al. The (CA)n polymorphism of ER $\beta$ gene is associated with FtM transsexualism. J Sex Med 2014;11:720-728.

21. Hengstschläger $M$, van Trotsenburg $M$, Repa $C$, Marton $E$, Huber JC, Bernaschek G. Sex chromosome aberrations and transsexualism. Fertil Steril 2003;79:639-640.

22. Schneider HJ, Pickel J, Stalla GK. Typical female 2nd-4th finger length (2D:4D) ratios in male-to-female transsexuals-possible implications for prenatal androgen exposure. Psychoneuroendocrinology 2006;31:265-269.

23. Kraemer B, Noll T, Delsignore A, Milos G, Schnyder U, Hepp $\mathrm{U}$. Finger length ratio (2D:4D) in adults with gender identity disorder. Arch Sex Behav 2009;38:359-363.

24. Zheng Z, Cohn MJ. Developmental basis of sexually dimorphic digit ratios. Proc Natl Acad Sci U S A 2011;108:1628916294.

25. Bentz EK, Hefler LA, Kaufmann U, Huber JC, Kolbus A, Tempfer CB. A polymorphism of the CYP17 gene related to sex steroid metabolism is associated with female-to-male but not male-to-female transsexualism. Fertil Steril 2008;90:5659.

26. Rodríguez-Larralde A, Paradisi I. [Influence of genetic factors on human sexual orientation. Review]. Invest Clin 2009;50: 377-391. Spanish.

27. Tu D, Xu R, Zhao G, Wang B, Feng T. [Research progress on molecular genetics of male homosexuality]. Zhonghua Yi Xue Yi Chuan Xue Za Zhi 2016;33:569-572. Chinese.

28. Lawrence AA, Zucker KJ. Gender dysphoria. In: Beidel DC, Frueh BC, Hersen M, editors. Adult psychopathology and diagnosis. 7th ed. Hoboken:John Wiley \& Sons;2014. p.603639.

29. Heylens G, Elaut E, Kreukels BP, Paap MC, Cerwenka S, Richter-Appelt $\mathrm{H}$, et al. Psychiatric characteristics in transsexual individuals: multicentre study in four European countries. Br J Psychiatry 2014;204:151-156.

30. Lin CS, Ku HL, Chao HT, Tu PC, Li CT, Cheng CM, et al. Neural network of body representation differs between transsexuals and cissexuals. PLoS One 2014;9:e85914.

31. Swaab DF, Garcia-Falgueras A. Sexual differentiation of the human brain in relation to gender identity and sexual orientation. Funct Neurol 2009;24:17-28.

32. Bao AM, Swaab DF. Sexual differentiation of the human brain: relation to gender identity, sexual orientation and neuropsychiatric disorders. Front Neuroendocrinol 2011;32: 214-226.

33. Zubiaurre-Elorza L, Junque $C$, Gómez-Gil E, Segovia $S$, Carrillo B, Rametti G, et al. Cortical thickness in untreated transsexuals. Cereb Cortex 2013;23:2855-2862. 
34. Luders E, Sánchez FJ, Tosun D, Shattuck DW, Gaser C, Vilain $\mathrm{E}$, et al. Increased cortical thickness in male-to-female transsexualism. J Behav Brain Sci 2012;2:357-362.

35. Abé C, Johansson E, Allzén E, Savic I. Sexual orientation related differences in cortical thickness in male individuals. PLoS One 2014;9:e114721.

36. Emory LE, Williams DH, Cole CM, Amparo EG, Meyer WJ. Anatomic variation of the corpus callosum in persons with gender dysphoria. Arch Sex Behav 1991;20:409-417.

37. Yokota Y, Kawamura Y, Kameya Y. Callosal shapes at the midsagittal plane: MRI differences of normal males, normal females, and GID. Conf Proc IEEE Eng Med Biol SOC 2005;3:3055-3058.

38. Rametti G, Carrillo B, Gómez-Gil E, Junque C, Segovia S, Gomez Á, et al. White matter microstructure in female to male transsexuals before cross-sex hormonal treatment. A diffusion tensor imaging study. J Psychiatr Res 2011;45:199-204.

39. Rametti G, Carrillo B, Gómez-Gil E, Junque C, ZubiarreElorza L, Segovia S, et al. The microstructure of white matter in male to female transsexuals before cross-sex hormonal treatment. A DTI study. I Psychiatr Res 2011;45:949-954.

40. Kranz GS, Hahn A, Kaufmann U, Küblböck M, Hummer A, Ganger $\mathrm{S}$, et al. White matter microstructure in transsexuals and controls investigated by diffusion tensor imaging. I Neurosci 2014;34:15466-15475.

41. Zhou JN, Hofman MA, Gooren LJ, Swaab DF. A sex difference in the human brain and its relation to transsexuality. Nature 1995;378:68-70.

42. Kruijver FP, Zhou JN, Pool CW, Hofman MA, Gooren LJ, Swaab DF. Male-to-female transsexuals have female neuron numbers in a limbic nucleus. J Clin Endocrinol Metab 2000; 85:2034-2041.

43. Garcia-Falgueras A, Swaab DF. A sex difference in the hypothalamic uncinate nucleus: relationship to gender identity. Brain 2008; 131:3132-3146.

44. Luders E, Sánchez FJ, Gaser C, Toga AW, Narr KL, Hamilton $\mathrm{LS}$, et al. Regional gray matter variation in male-to-female transsexualism. Neuroimage 2009;46:904-907.

45. Savic I, Arver S. Sex dimorphism of the brain in male-to-female transsexuals. Cereb Cortex 2011;21:2525-2533.

46. Simon L, Kozák LR, Simon V, Czobor P, Unoka Z, Szabó Á, et al. Regional grey matter structure differences between transsexuals and healthy controls--a voxel based morphometry study. PLoS One 2013;8:e83947.

47. Hahn A, Kranz GS, Küblböck M, Kaufmann U, Ganger S, Hummer A, et al. Structural connectivity networks of transgender people. Cereb Cortex 2015;25:3527-3534.

48. Santarnecchi E, Vatti G, Déttore D, Rossi A. Intrinsic cerebral connectivity analysis in an untreated female-to-male transsexual subject: a first attempt using resting-state fMRI.
Neuroendocrinology 2012;96:188-193.

49. Berglund H, Lindström P, Dhejne-Helmy C, Savic I. Male-tofemale transsexuals show sex-atypical hypothalamus activation when smelling odorous steroids. Cereb Cortex 2008;18: 1900-1908.

50. Burke SM, Cohen-Kettenis PT, Veltman DJ, Klink DT, Bakker J. Hypothalamic response to the chemo-signal androstadienone in gender dysphoric children and adolescents. Front Endocrinol (Lausanne) 2014;5:60.

51. Ku HL, Lin CS, Chao HT, Tu PC, Li CT, Cheng CM, et al. Brain signature characterizing the body-brain-mind axis of transsexuals. PLoS One 2013;8:e70808.

52. Gizewski ER, Krause E, Schlamann M, Happich F, Ladd ME, Forsting $\mathrm{M}$, et al. Specific cerebral activation due to visual erotic stimuli in male-to-female transsexuals compared with male and female controls: an fMRI study. I Sex Med 2009;6: 440-448.

53. Soleman RS, Staphorsius AS, Cohen-Kettenis PT, Lambalk CB, Veltman DJ, van Trotsenburg MA, et al. Oestrogens are not related to emotional processing: a study of regional brain activity in female-to-male transsexuals under gonadal suppression. Cereb Cortex 2016;26:510-516.

54. Junger J, Habel U, Bröhr S, Neulen J, Neuschaefer-Rube C, Birkholz P, et al. More than just two sexes: the neural correlates of voice gender perception in gender dysphoria. PLoS One 2014;9:e111672.

55. Schöning S, Engelien A, Bauer C, Kugel H, Kersting A, Roestel $\mathrm{C}$, et al. Neuroimaging differences in spatial cognition between men and male-to-female transsexuals before and during hormone therapy. I Sex Med 2010;7:1858-1867.

56. Soleman RS, Schagen SE, Veltman DJ, Kreukels BP, CohenKettenis PT, Lambalk CB, et al. Sex differences in verbal fluency during adolescence: a functional magnetic resonance imaging study in gender dysphoric and control boys and girls. J Sex Med 2013;10:1969-1977.

57. Staphorsius AS, Kreukels BP, Cohen-Kettenis PT, Veltman DJ, Burke SM, Schagen SE, et al. Puberty suppression and executive functioning: An fMRl-study in adolescents with gender dysphoria. Psychoneuroendocrinology 2015;56:190-199.

58. Han S, Ma Y. A culture-behavior-brain loop model of human development. Trends Cogn Sci 2015; 19:666-676.

59. Han S. Understanding cultural differences in human behavior: a cultural neuroscience approach. Curr Opin Behav Sci 2015;3:68-72.

60. Han S, Ma Y. Cultural differences in human brain activity: a quantitative meta-analysis. Neuroimage 2014;99:293-300.

61. Han S, Northoff G, Vogeley K, Wexler BE, Kitayama S, Varnum ME. A cultural neuroscience approach to the biosocial nature of the human brain. Annu Rev Psychol 2013;64: 335-359. 